\title{
Small Radio Telescopes for Education
}

\author{
Koitiro Maeda \\ Department of Physics, Hyogo College of Medicine Nishinomiya, Hyogo \\ 663-8501, Japan. e-mail:maeda@hyogomc.kugi.kyoto-u.ac.jp \\ Noritaka Tokimasa \\ Nishi-harima Astronomical Observatory Sayo-cho, Hyogo 679-5313, \\ Japan
}

\begin{abstract}
We present small radio telescopes consisting of commercial instruments for satellite TV reception. With these radio telescopes we can observe the $12 \mathrm{GHz}$ emissions from the quiet sun and solar flares. Since microwaves occurring in our environment, e.g., those from a building and a fluorescent lamp, are also detectable, such radio telescopes are useful not only for radio astronomy education but also for physics education.
\end{abstract}

\section{Introduction}

At low frequencies (HF and VHF bands), the galactic background radiation, solar bursts, and Jupiter's decametric radiation are strong and detectable with a radio telescope consisting of a simple antenna (e.g., a dipole or a 3-element Yagi) and a commercial communications receiver. Maeda (1990a) reported such simple radio telescopes to observe the galactic background radiation and solar bursts at about $30 \mathrm{MHz}$. Since the thermal emission from the quiet Sun is relatively weak at low frequencies, some sensitive observations are necessary to detect it. Phase-switched interferometers designed for education were used to observe the quiet Sun, Cas A, Cyg A, and Tau A at $74 \mathrm{MHz}$ (Swenson, 1978) and at $49.5 \mathrm{MHz}$ (Maeda $1990 \mathrm{~b}$ ). The quiet-Sun component becomes stronger with increasing frequency. Radio telescopes for the microwave region have been largely beyond the reach of teachers and amateurs, owing to the high cost of antenna with a large collecting area. Now, however, we can do some interesting astronomy with widely available antennas with sensitive electronics designed for satellite TV reception. The mapping of the Milky Way at a wavelength of $21 \mathrm{~cm}$ was made using such an antenna (Schuler, 1994; Rogers, 1996). In this paper we describe the small radio telescopes that are portable and usable for observing the sun at $12 \mathrm{GHz}$.

\section{Small Radio-Telescope at $12 \mathrm{GHz}$}

The small radio telescope that we made consists of a commercial satellite TV antenna with an effective diameter of $35 \mathrm{~cm}$, a commercial IF booster, a homemade detector, and a voltage-measuring device (see Figure 1). The satellite TV 


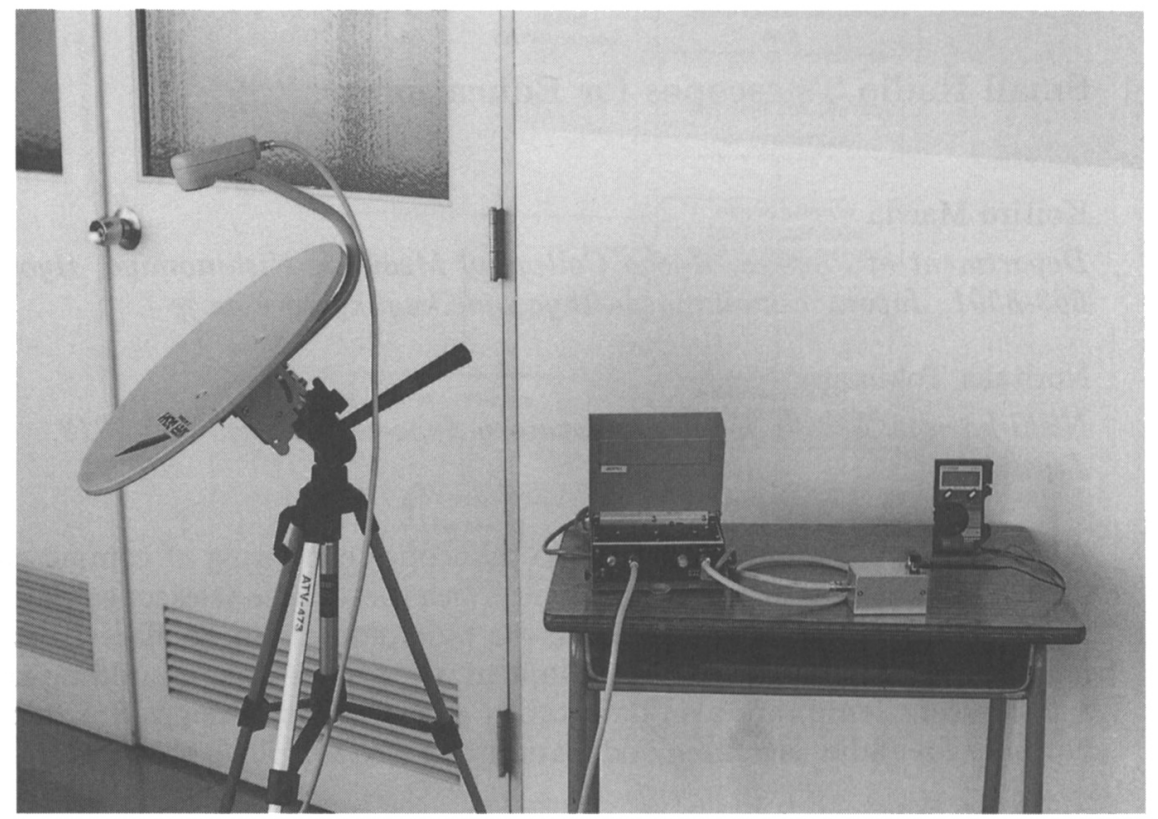

Figure 1. The simplest radio-telescope we made. The satellite TV antenna of $35 \mathrm{~cm}$ effective diameter mounted on a video-camera tripod is at the far left. On the small desk, from left to right an IF booster, a home-made detector, and a digital multi-meter are seen.

antenna we used consists of an offset parabolic reflector and a frequency converter. This antenna receives the right-hand circularly polarized signal. The antenna is mounted on a video-camera tripod using a cylindrical aluminum bar. The antenna beam direction is therefore easily changeable. Since the effective diameter of the antenna is $35 \mathrm{~cm}$, the beamwidth at $12 \mathrm{GHz}$ (a wavelength of $2.5 \mathrm{~cm}$ ) is about 4 degrees. The nominal antenna gain is about $32 \mathrm{~dB}$. The frequency converter amplifies the received $12-\mathrm{GHz}$ signal by $52 \mathrm{~dB}$ and outputs an IF signal of $1 \mathrm{GHz}$. The noise figure of the converter is $0.9 \mathrm{~dB}$. The $1-\mathrm{GHz}$ signal from the converter is fed into the IF booster and further amplified by about $30 \mathrm{~dB}$. Such a booster is commonly used for satellite TV reception at an apartment and commercially obtainable. The amplified IF signal is detected to get a D.C. voltage. We use a simple homemade detector. A commercial detector is also available. The output D.C. voltage of the detector is about several tens of millivolts and measurable with a digital multi-meter. For obtaining a continuous record, a pen recorder is useful. It is also good for students to use an analog-digital converter to graphically display the data on a personal computer.

\section{Observation of the Sun}

The beamwidth of the antenna is about $4^{\circ}$, that is much greater than the angular radius of the Sun, i.e., $0.5^{\circ}$. It is relatively easy to catch the Sun even in a cloudy 


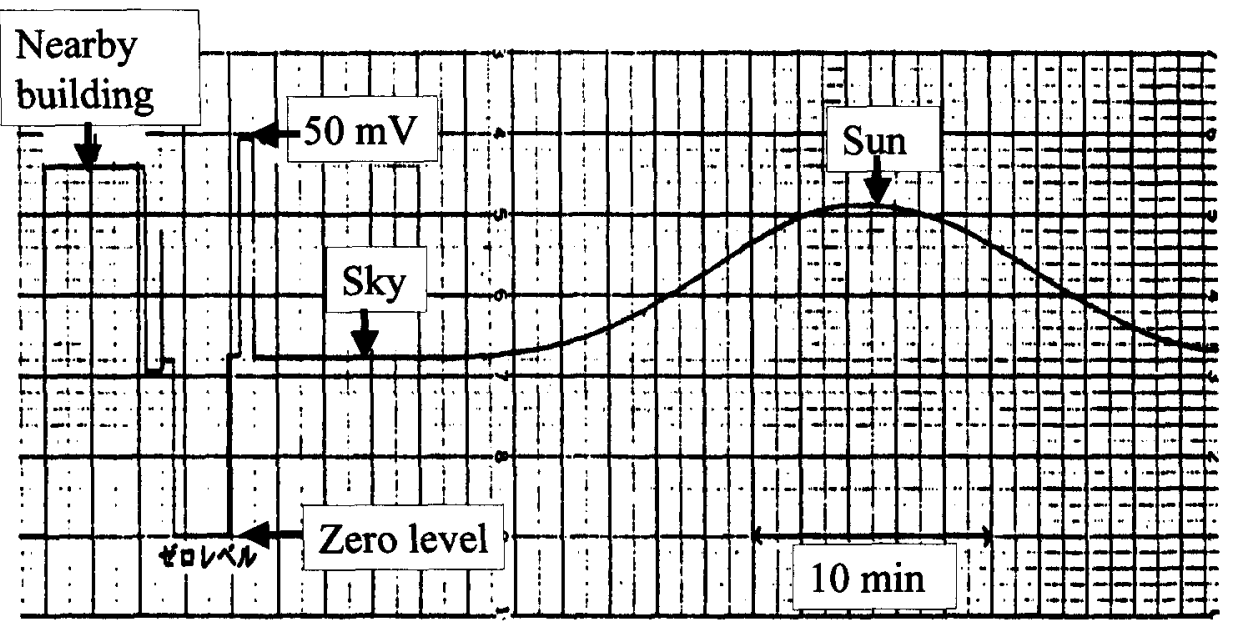

Figure 2. Drift-scan of the Sun recorded on a pen-recorder. The satellite TV antenna having an effective diameter of $45 \mathrm{~cm}$ was used. The levels of the sky and a nearby building are also shown.

sky. On a sunny day the sunlight can be used for pointing. A good way is to put a piece of well reflective tape on the parabolic reflector surface. The reflected light from the tape makes a bright spot on the converter input surface. By setting the bright spot at the center of the converter input surface we can easily point the antenna beam at the Sun. The sky background at $12 \mathrm{GHz}$ consists of the cosmic background radiation of $2.7 \mathrm{~K}$ and the atmospheric emission (say, about 3 $\mathrm{K})$. A simple calibration method was tested using the sky background radiation and the thermal radiation of the wall of a room. By a linear interpolation the antenna temperature due to the sun was estimated to be about $130 \mathrm{~K}$. The flux density was calculated using this antenna temperature and the effective area of the antenna. We obtained a flux density value of $4.8 \times 10^{6} \mathrm{Jy}$. This value is in good agreement with that given in the textbook of Kruger (1979). Figure 2 shows an example of the drift scan observation of the sun recorded on a pen recorder. The antenna of $45 \mathrm{~cm}$ effective diameter was used for this observation.

\section{An Advanced Version of the Small Radio-Telescope}

Figure 3 shows an advanced version of the small radio telescope. The satellite TV antenna of the effective diameter of $45 \mathrm{~cm}$ is used for this radio telescope. The gain and beamwidth of this antenna are about $34 \mathrm{~dB}$ and 3.2 degrees, respectively. The satellite TV antenna is set on the equatorial mount that is commonly used for an optical telescope, using a joint as shown in Figure 3. By tracking the Sun and detecting solar bursts we can do solar flare patrol. Since the solar flare affects the terrestrial upper atmosphere in various ways, such an observation is useful for education of the upper atmospheric environment, so called space weather. 


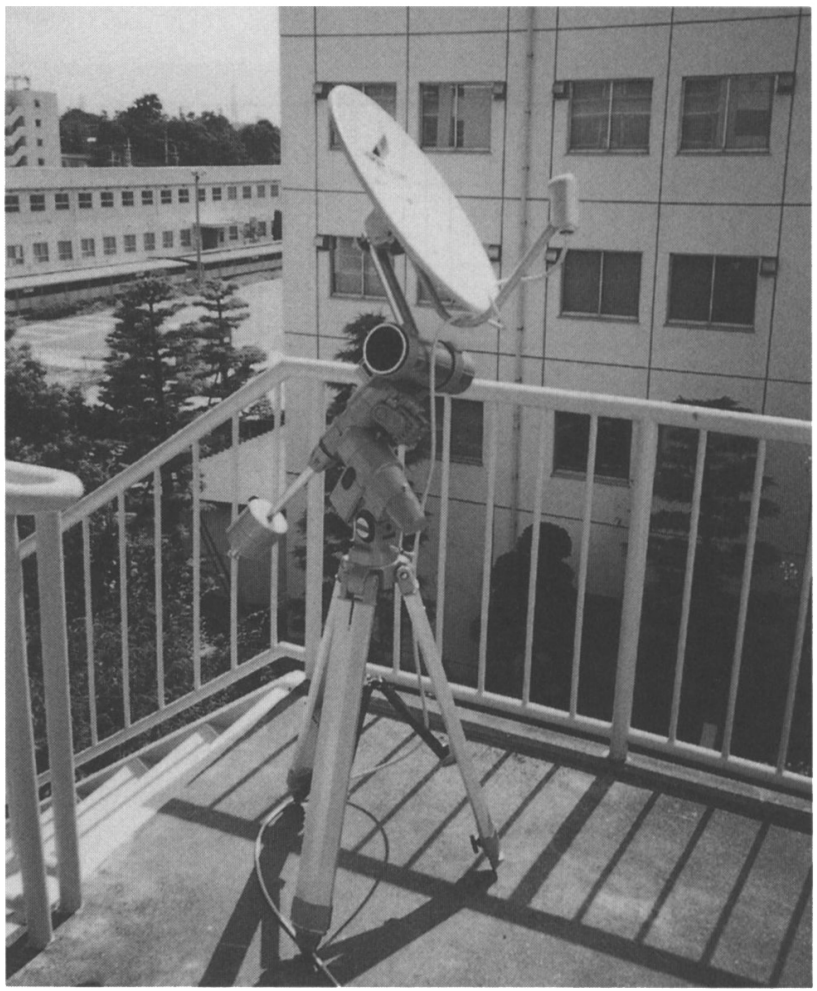

Figure 3. An advanced version of the small radio-telescope. The satellite TV antenna, its effective diameter being $45 \mathrm{~cm}$, is set on an equatorial mount for an optical telescope.

\section{Other Experiments with the Small Radio-Telescope}

At $12 \mathrm{GHz}$ everything in our environment radiates radio waves. The human body is emitting the microwaves as thermal radiation at a temperature of about $310 \mathrm{~K}$, which is much greater than that of the noise temperature of the sky. We can easily show the human body is really emitting the microwaves using the small radio telescope. Since it is easy to carry the small radio telescope, we can use it for a lecture in a classroom. In this respect a fluorescent lamp is useful as an artificial radio source. The directivity of the antenna is easily demonstrated using the fluorescent lamp. By blocking the ray path of the radio waves by various materials we can demonstrate what kind of material effectively attenuates the microwaves. An interferometer experiment is also possible using a fluorescent lamp and an aluminum plate as a reflector. We therefore believe that small radio telescopes are widely usable at various levels of physics education. 


\section{References}

Kruger, A., 1979, Introduction to Solar Radio Astronomy and Radio Physics, Reidel, Dordrecht, The Netherlands.

Maeda, K., 1990a, Sky \& Telescope 80, (August), 200.

Maeda, K., 1990b, Astronomical Herald, 83, 72. (in Japanese)

Rogers, A.E.E., 1996, Sky \& Telescope 92, (August), 75.

Schuler, P.W. III, 1994, Sky \& Telescope 87, (March), 91.

Swenson, G.W., 1978, Sky \& Telescope 56, (October), 290. 